\title{
Energy generation rate densities of ultrahigh-energy cosmic-ray nuclei
}

\author{
Yu Jiang ${ }^{* a b c}$ B.Theodore Zhang ${ }^{d e a}$ and Kohta Murase ${ }^{a b c f}$ \\ ${ }^{a}$ Department of Physics, The Pennsylvania State University \\ University Park, Pennsylvania 16802, USA \\ ${ }^{b}$ Department of Astronomy \& Astrophysics, The Pennsylvania State University \\ University Park, Pennsylvania 16802, USA \\ ${ }^{c}$ Center for Particle and Gravitational Astrophysics, The Pennsylvania State University \\ University Park, Pennsylvania 16802, USA \\ ${ }^{d}$ Department of Astronomy, School of Physics, Peking University \\ Beijing 100871, China \\ ${ }^{e}$ Kavli Institute for Astronomy and Astrophysics, Peking University \\ Beijing 100871, China \\ ${ }^{f}$ Yukawa Institute for Theoretical Physics, Kyoto \\ Kyoto 606-8502 Japan \\ E-mail: jiang@psu.edu, uni $00011011 @ g m a i l . c o m, m u r a s e @ p s u . e d u$
}

The origin of ultrahigh-energy cosmic-ray (UHECR) has been a mystery for many years. In order to identify candidate sources, it is crucial to figure out the energetics of cosmic-ray sources. By fitting the experiment data of cosmic-ray energy spectrum from Pierre Auger Observatory, we calculate the energy generation rate densities for different nuclei species via scanning over various parameters that are related to the source distribution models, injection properties as well as propagation of UHECR. Assuming UHECRs are protons and heavy nuclei produced by extragalactic sources, the energy generation rate density is estimated to be $\varepsilon^{2} Q \sim 0.66_{-0.3}^{+0.8} \times 10^{44}$ erg $\mathrm{Mpc}^{-3} \mathrm{yr}^{-1}$ averaging different nuclei species at power-law spectral index $s=2.0$ and it varies on different fitting range. For a power-law scaling of the $\mathrm{CR}$ production rate with redshift, maximum energy and pure proton injection, $d N / d E \propto E^{-s} \exp \left(-E / E_{\max }\right)(1+z)^{m}$, best-fit parameters are $s=2.1, m=5.0, E_{\max }=10^{19.9} \mathrm{eV}$. Hard spectra with strong source redshift evolution and low maximal source energy are slightly favored. We did not find a strong proof of the linear relationship between $\varepsilon^{2} Q$ and $s$ as shown in Katz et al 2009. We discuss cases of mixed composition models by analyzing the combined fit of spectrum and mass composition $X_{\max }$ data.

36th International Cosmic Ray Conference -ICRC2019-

July 24th - August 1st, 2019

Madison, WI, U.S.A.

* Speaker. 


\section{Introduction}

Ultrahigh-energy cosmic rays (UHECRs) are charged particles of astrophysical origin with energies above $10^{9} \mathrm{GeV}$, the highest observed in the Universe so far. Although world-wide experiments and great effort put into detecting and understanding this interesting phenomenon, their origin remains unknown $[1,2,3]$. Pierre Auger Observatory is one of the largest cosmic ray experiment that collects data towards investigating the nature and origin of UHECRs [4].

The spectrum of UHECRs flattens around $5 \times 10^{18} \mathrm{eV}$ (the "ankle"), and there is a strong suppression at the topmost energies, around $5 \times 10^{19} \mathrm{eV}$ [5]. The formation of the "ankle" can be explained naturally by the transition from galactic origin to extragalactic origin. The sharp decline of the flux of UHECRs beyond $5 \times 10^{19} \mathrm{eV}$ is consistent with the predictions of Greisen-ZatespinKuzmin (GZK) cutoff $[6,7]$ where UHECRs lose energy by the interaction with CMB photons during their propagation in the intergalactic space, or caused by the limited maximum acceleration energy inside the sources [1]. The composition of cosmic rays at ultra-high energies is crucial for us to understand their origin.

There have been many efforts have been done in order to unveil the mysterious of UHECRs, see Ref. [3] for a recent review. In this work, we aim to give constraints on the UHECRs energy generation rate density via considering UHECRs are composed by various nuclei species.

\section{Method}

The sources of UHECRs and its acceleration process are still unclear. There are many acceleration mechanisms being studied previously. These include the first order Fermi shock acceleration [8], plasma wakefield acceleration [9] and so on. Among these models, the accelerated UHECRs usually have power-law spectra $d N / d E \propto E^{-s}$ with exponential cut-off at the highest energy. In some mechanisms, $s<0$, while the more meaningful ones range from 1.0 to $2.0[10,11,12]$. The basic assumption we take is that the sources are identical and their distribution is isotropic and uniform in the universe. Thus, it is adequate to consider 1D propagation in this work, where the sources redshift ranging from $z_{\min }=0.0001$ to $z_{\max }=2.1$. As a result, we assume a distribution of identical sources with one species of nuclei injection [13] $J^{i n j}(E) \propto F(z) E^{-s} \exp \left(-E / E_{\max }\right)$, where $E$ is the energy of the injected nuclei and $F(z) \propto(1+z)^{m}$ is assumed to implement the redshift evolution of the number density of the sources.

When UHECR nuclei are propagated in the intergalactic space, not only their energy will be lost, but also the heavy nuclei might be broken into small pieces due to the photodisintegration process. Other energy loss processes include photopion production, Bethe-Heitler pair production processes, and adibatic energy loss processes. In addition to the cosmic microwave background radiation (CMB), we also consider the extragalactic backgroung light (EBL) [14], which is important for the photodisintegration of UHECR nuclei at slightly lower energy.

We utilize the numerical code CRPropa 3 to propagate UHECR nuclei through the universe, until they are detected at Earth. In order to fit the observation data, we treat following parameters as free parameters, including spectral index $s$, maximum acceleration energy $E_{\max }$, redshift evolution index $m$. 
We use the chi-square method to estimate the goodness of fitting of the observed energy spectrum [13].

$$
\chi_{\text {spec }}^{2}=\sum_{i} \frac{\left(f J^{\operatorname{sim}}\left(E_{i}^{\prime} ; s, E_{\max }, m\right)-J^{\text {Auger }}\left(E_{i}\right)\right)^{2}}{\sigma_{i}^{2}}+\left(\frac{\delta_{E}}{\sigma_{E}}\right)^{2},
$$

where $f$ is the free normalization factor, $J^{\operatorname{sim}}\left(E_{i}^{\prime} ; s, E_{\max }, m\right)$ is the simulated flux at Earth, $J^{\text {Auger }}\left(E_{i}\right)$ is the UHECRs flux measured by Auger, $\sigma_{i}$ contains both systematic and statistic error, $E_{i}^{\prime} \equiv\left(1+\delta_{E}\right) E_{i}$ takes into account the energy uncertainty on measured UHECR nuclei, $\sigma_{E}=14 \%$ is the systematic uncertainty on the measured energy scale.

If we have the knowledge of mass number $A$ and energy $E$ of primary particles, we are able to estimate the distribution of $X_{\max }$ using certain hadronic interaction models. In other words, after simulating propagation of UHECR, the mass composition reaching Earth can be transformed into consequential $X_{\max }$ distribution if we adopt one of hadronic interaction models (EPOS-LHC, Sibyll 2.1, QGSJet II-04 and etc.). We are not simulating the realistic air showers or cascade process. We use generalized Gumbel distribution function [15] to describe the underlying probability density function (p.d.f.) of $X_{\max }$, in which there are $E$ and $A$ dependent parameters $\mu, \sigma, \lambda$ as well as parameters $a, b, c$ obtained from CONEX [16] shower simulations that are based on different UHE nuclei hadronic interaction models. After summing up the result of different species of nuclei with a weight of their percentage in each energy bin, we can calculate the final simulated $X_{\max }$ distribution outcome that can be compared to the observables, namely $\left\langle X_{\max }>\right.$ and $\sigma\left(X_{\max }\right)$, from experiment [17].

Similar to spectral fit, we use the chi-square method to estimate the goodness of $\left\langle X_{\max }>\right.$ and $\sigma\left(X_{\max }\right)$ fit. In addition, we can also derive the combined fitting considering spectrum, $\left\langle X_{\max }\right\rangle$ and $\sigma\left(X_{\max }\right)$. Of course, considering the full information of $X_{\max }$ distributions rather than their first two statistical moments would yield more accurate result and avoid possible coincidence of $<X_{\max }>$ and $\sigma\left(X_{\max }\right)$ out of different distributions [17]. That might be the reason of the divergence between our result and these by Auger's analysis [18]. A uniform scan over four dimensional grid $\left(s, \log _{10}\left(E_{\max }\right), m, \delta_{E}\right)$ is performed, and on each grid point, the discrepancy between simulated and experimental data is a function of the energy generation rate density at the source. The bestfit is when the discrepancy is minimized as it means. The experimental data set to be fit with simulation result have two parts: the event distribution in energy bins of 0.1 in $\log _{10}(E / \mathrm{eV})$, and $X_{\max }$ distribution in the same energy bins up to $\log _{10}(E / \mathrm{eV})=19.5$ and final bin combining data $\log _{10}(E / \mathrm{eV})$ from 19.5 to 20.0. We use CRPropa 3 [19] to simulate the propagation of UHECRs through the universe.

\section{Results of spectral fits}

In this section, we present the fitting results considering spectral data only. The best-fit parameters for pure proton injection are $\log _{10}\left(E_{\max }\right)=19.9, s=2.1, m=5.0, \delta_{E}=0.06$ and $\chi^{2} /$ d.o.f $=$ 0.779 . We can see that the spectral fit is quite well as the value of $\chi^{2} /$ d.o.f shows, which corresponds to a p-value of $71.9773 \%$ and is not significant at all. As shown in Figure 1, if we only look into how energy generation rate density changes with power-law index $s$ and let other parameters to optimize $\chi^{2}$, the result more or less obey the analytic analysis result by Ref. [20], which stated that 
energy generation rate $\varepsilon^{2} Q\left(\varepsilon>10^{19.5} \mathrm{eV}\right) \sim 0.3-0.6 \times 10^{44} \times(s-1) \mathrm{erg}^{\mathrm{Mpc}} \mathrm{pr}^{-1} \mathrm{yr}^{-1}$. However, opposite to previous claim, the linear relation between energy generation rate density and $s$ when $2.0<s<2.7$ is not solid in our result. Of course, the result changes when we change the fitting energy range, see the right panel of Figure 1 . Fewer data points to be fit make smaller $\chi^{2} /$ d.o.f possible. Generally, the correlations between parameters look similar.

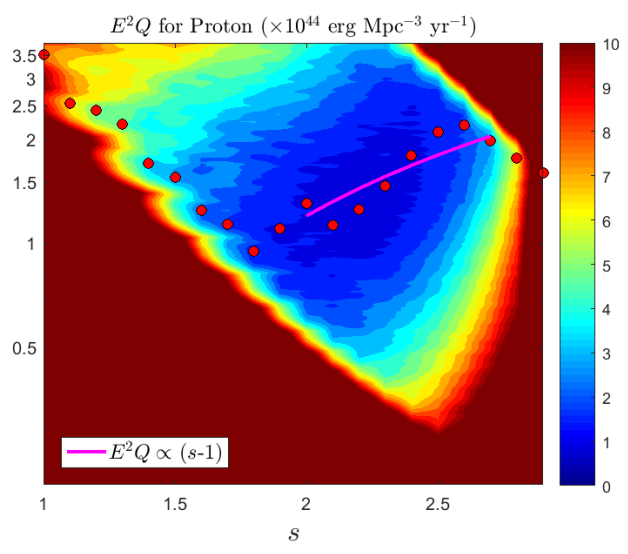

(a) Fitting energy range $10^{18.45} \mathrm{eV}$ to $10^{20.15} \mathrm{eV}$

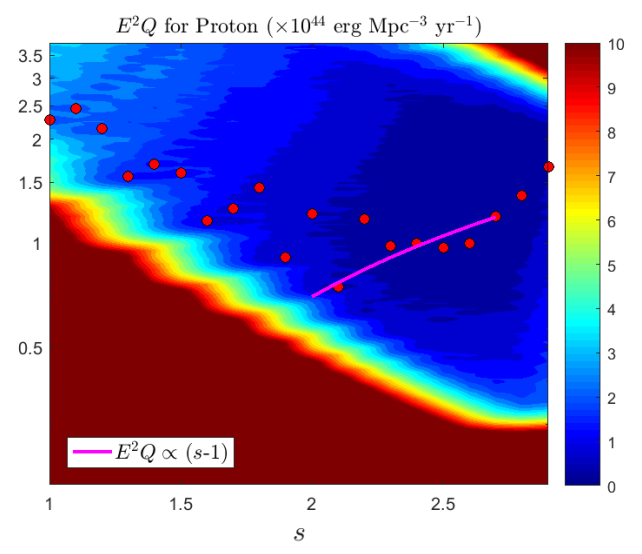

(b) Fitting energy range $10^{19.05} \mathrm{eV}$ to $10^{20.15} \mathrm{eV}$

Figure 1: Best-fit energy generation rate density $E^{2} Q$ as a function of spectral power-law index $s$, compared with analytic result $E^{2} Q \propto s-1$ in the range of [2.0,2.7]. $m$ and $E_{\max }$ are scanned. The background contour reflects the minimal $\chi^{2} /$ d.o.f with different combinations of energy generation rate density and power-law index $s$. Pure proton is injected at the source. Subplots show results from different fitting energy range.

Some interesting and evident features emerge in the 3D scan (over $s, \log _{10}\left(E_{\max }\right), m$ ) of parameters (allow all parameters, including energy generation rate density $E^{2} Q$, change freely to find the minimal $\left.\chi^{2}\right)$. In Figure 2 we present the minimal $\chi^{2} /$ d.o.f value as a function of $(s, m)$ and $\left(E_{\max }, s\right)$. The distribution of minimal $\chi^{2} /$ d.o.f, corresponding to the best fit, behave like valley curves. For region $s<2.0$, the minimal $\chi^{2} /$ d.o.f is not low enough for a good fitting, especially when we require a low redshift revolution $m(<5.0)$ or high $E_{\max }\left(>10^{20.0} \mathrm{eV}\right)$. There are certain regions of parameters that can give reasonable good fitting $\left(\chi^{2} /\right.$ d.o.f $<2$, corresponding to a p-value of $0.8396 \%)$. Actually, these regions show clear correlations among the parameters we searched $\left(s, E_{\max }, m\right)$, and that is the case for different species of nuclei and their mixed injection as we show later. For instance, when $m$ gets smaller, higher $s$ is required in order to produce low $\chi^{2}$. However, these general features are not exactly the same for different nuclei. For example, in the case of proton, there is one single minimum for the $\chi^{2} /$ d.o.f, while for some heavier nuclei, there are more than one local minima, which means that there are multiple regions with good fit but are not adjacent to each other.

For pure injection of light and intermediate nuclei, the spectral fits can also be good ( $\mathrm{He}, \mathrm{C}, \mathrm{Si}$ and so on). The minimal $\chi^{2} /$ d.o.f $=0.938,1.112,1.425,1.449$ and 1.238 for $\mathrm{He}, \mathrm{C}, \mathrm{N}, \mathrm{O}$ and $\mathrm{Si}$ injection respectively. Comparing to the case of Proton injection, heavier nuclei injection generally do not yield spectral fit as good as Proton, with larger $\chi^{2} /$ d.o.f, especially for large nuclear mass A. 


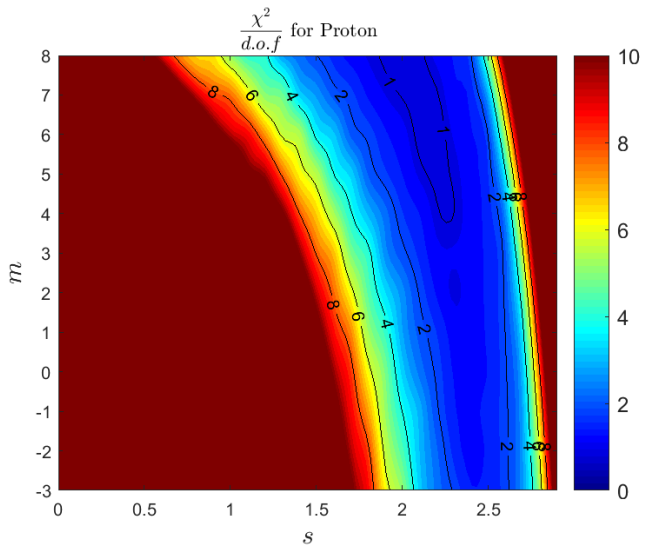

(a) minimal $\chi^{2} /$ d.o.f as a function of powerlaw index $s$ and redshift evolution $m . E_{\max }$ and $\delta_{E}$ are scanned.

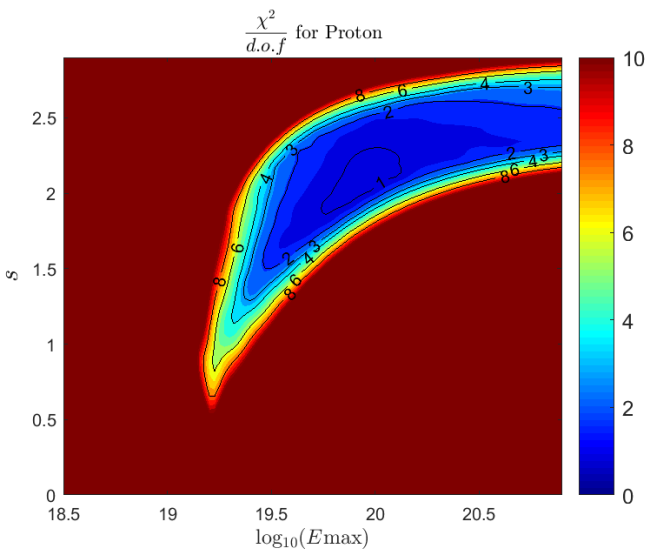

(b) minimal $\chi^{2} /$ d.o.f as a function of powerlaw index $s$ and $E_{\max }$. Redshift evolution $m$ and $\delta_{E}$ are scanned.

Figure 2: 3D parameter scan of pure proton injection. The solid contour indicates certain values of $\chi^{2} /$ d.o.f., smaller of which means better fit.

We covered $\mathrm{Fe}$ as the heaviest nuclei injection. It does not have a very good spectral fit with minimal $\chi^{2} /$ d.o.f $=2.567$. In [18] composition analysis that can lead to best-fit, Fe fraction was zero and excluded from further discussion.

Last but not least, after testing all above different species of nuclei from light to heavy, we have a plot of energy generation rate density with minimal $\chi^{2}$ as a function of injected atomic mass in Figure 3. The error bars represent the range of generation rate density that can lead to a spectral fit with $\chi^{2} /$ d.o.f $<3$, which corresponds to a p-value of $0.002951 \%$.

\section{Results of spectral and composition fits}

In this section, we consider the combined fit of both spectrum and composition. The fitting range for $\left\langle X_{\max }\right\rangle$ and $\sigma\left(X_{\max }\right)$ is from $10^{18.45} \mathrm{eV}$ to $10^{19.65} \mathrm{eV}$. Si, for instance, the parameter set for its spectral best fit can give $<X_{\max }>$ and $\sigma\left(X_{\max }\right)$ data a good fit as well. That means adding fitting $<X_{\max }>$ and $\sigma\left(X_{\max }\right)$ will not change the best-fit parameters much compared to only fitting spectrum, and some regions in parameter space can have small total $\chi^{2}$ (thus good fit) combining spectral and $X_{\max }$ fit as shown in Figure 4. However, for some other nuclei, the spectral best fit parameter set give poor fit to $\left\langle X_{\max }\right\rangle$ and $\sigma\left(X_{\max }\right)$ data. For example, $\mathrm{O}$ and Fe. There is no such a region in the parameters space that have a small total $\chi^{2}$, which means that it is impossible to have good spectral and $X_{\max }$ fit with same parameter set. Actually, among these injected nuclei examined, only Si can produce a decent fitting of $X_{\max }$ data when considering spectrum and $X_{\max }$ together for the best-fit parameters. This is also the case in [18]: absence of Si injection would make the fitting of spectrum much worse when considering both spectrum and $X_{\max }$ for the best-fit.

\section{Summary and discussion}

Under the assumption that the UHECRs have extragalactic origins, we estimate the UHECR energy generation rate density in the case of different nuclei injections by fitting experimental 


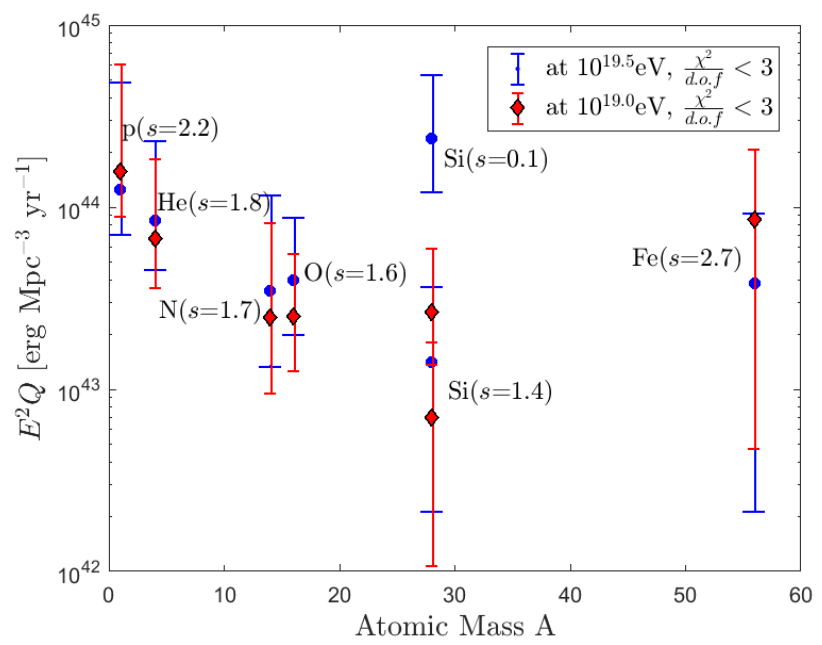

Figure 3: The UHECR energy generation rate density with minimal $\chi^{2}$ as a function of the mass of injected nuclei, where we consider several typical nuclei species, such as protons, $\mathrm{He}, \mathrm{N}, \mathrm{O}, \mathrm{Si}$ and Fe. Parameters $s, m, E_{\max }$ and $\delta_{E}$ are scanned. The energy generation rate densities range that can yield $\chi^{2} /$ d.o.f $<3$ fitting are showed as the error bar. The Si has two local minimal $\chi^{2}$ region, so as reflected in the plot.

measurements of UHECR spectrum and mass composition ( $X_{\max }$ statistical parameters).

The best-fit parameters indicate that hard injection fluxes, strong redshift evolution and low maximal energy at the sources give a better explanation for the Auger experimental spectrum, which is in line with the analysis from Auger [18]. At least that is the case for pure proton injection, when the fitting is overall good (with low $\chi^{2}$ ) and none of the fitting request too large systematic shift of the energy scale $\left(\delta_{E}<0.07\right)$. Assuming UHECRs are extragalactic protons, the energy generation rate density is $\varepsilon^{2} Q \sim 0.6-2.0 \times 10^{44} \mathrm{erg} \mathrm{Mpc}^{-3} \mathrm{yr}^{-1}$ depending on the fitting range [see Figure 1]. The results for heavier nuclei are summarized in Figure 3. Many previous work shared similar results, such as in Ref. [21, 22, 23].

In general, we are able to reproduce the received spectrum of UHECR with straightforward models and simple hypotheses in source features and propagation. On the other hand, the parameters in the models are constrained in order to generate a good spectral fit. With an appropriate choice of hadronic interaction model in the atmosphere of Earth, composition data is not well fitted considering different species of nuclei. With the following AugerPrime operation [24] and new experiments, other observables related to the composition as well as more detections will become available, and the systematic error will then be reduced in an more extended energy scale. This will help constrain source models and have implications for the origins.

\section{Acknowledgments}

This work is supported by the Alfred P. Sloan Foundation and NSF grant No. PHY-1620777 (K.M.). 


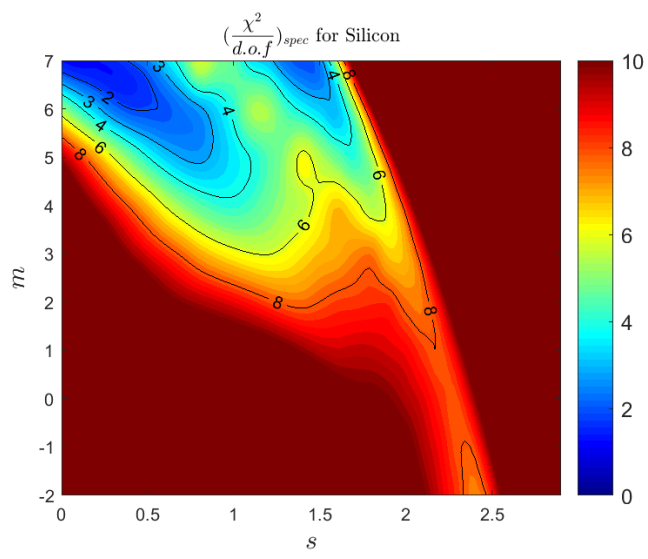

(a) $\chi^{2}$ from spectral fit only

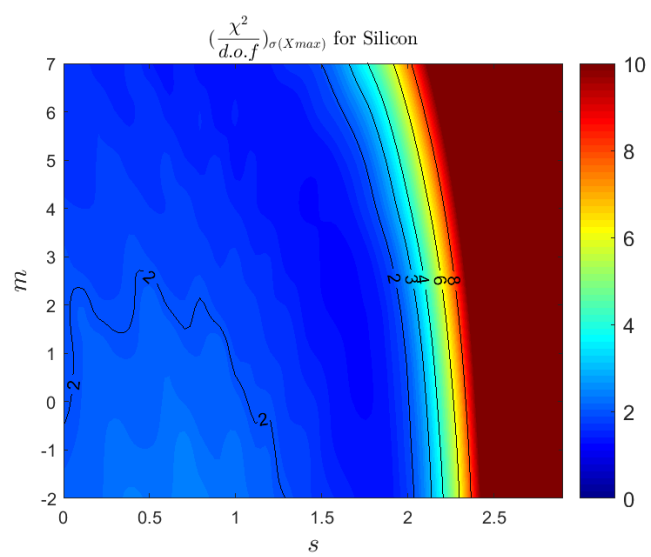

(c) $\chi^{2}$ from $\sigma\left(X_{\max }\right)$ fit only

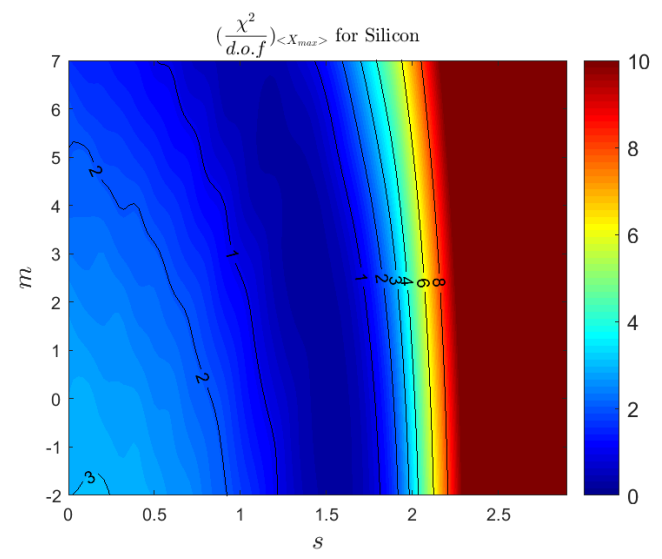

(b) $\chi^{2}$ from $<X_{\max }>$ fit only

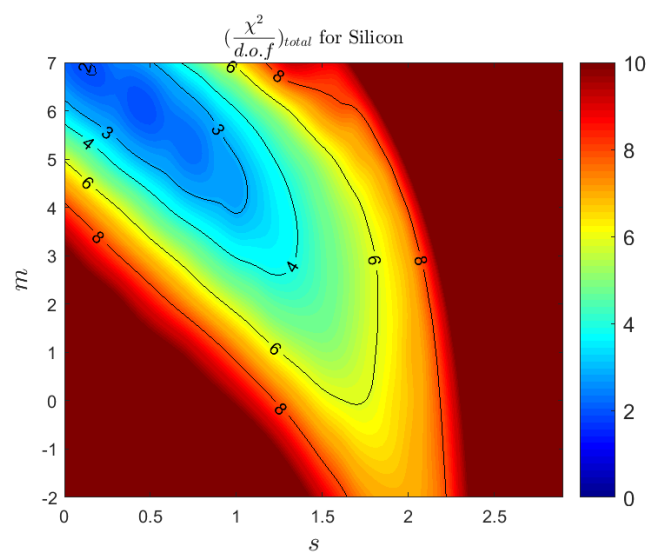

(d) total $\chi^{2}$ from spectral and two moments of $X_{\max }$ fits

Figure 4: Minimal $\chi^{2} /$ d.o.f as a function of power-law index $s$ and redshift evolution $m$. $E_{\max }$ and $\delta_{E}$ are scanned. Pure Si simulated injection.

\section{References}

[1] K. Kotera and A. V. Olinto, The Astrophysics of Ultrahigh Energy Cosmic Rays, Ann. Rev. Astron. Astrophys. 49 (2011) 119 [1101.4256].

[2] L. A. Anchordoqui, Ultra-High-Energy Cosmic Rays, 1807.09645.

[3] R. Alves Batista et al., Open Questions in Cosmic-Ray Research at Ultrahigh Energies, 1903.06714.

[4] Pierre Auger collaboration, The Pierre Auger Cosmic Ray Observatory, Nucl. Instrum. Meth. A798 (2015) 172 [1502.01323].

[5] Pierre Auger Collaboration collaboration, The Pierre Auger Observatory: Contributions to the 35th International Cosmic Ray Conference (ICRC 2017), in Proceedings, 35th International Cosmic Ray Conference (ICRC 2017): Bexco, Busan, Korea, July 12-20, 2017, 2017, 1708 . 06592.

[6] K. Greisen, End to the cosmic ray spectrum?, Phys. Rev. Lett. 16 (1966) 748.

[7] G. T. Zatsepin and V. A. Kuzmin, Upper limit of the spectrum of cosmic rays, JETP Lett. 4 (1966) 78. 
[8] A. Bykov, N. Gehrels, H. Krawczynski, M. Lemoine, G. Pelletier and M. Pohl, Particle acceleration in relativistic outflows, Space Sci. Rev. 173 (2012) 309 [120 5 .2208].

[9] P. Chen, T. Tajima and Y. Takahashi, Plasma wakefield acceleration for ultrahigh-energy cosmic rays, Phys. Rev. Lett. 89 (2002) 161101 [astro-ph/ 0205287 ].

[10] P. Blasi, R. I. Epstein and A. V. Olinto, Ultrahigh-energy cosmic rays from young neutron star winds, Astrophys. J. 533 (2000) L123 [astro-ph/9912240].

[11] K. Kotera, E. Amato and P. Blasi, The fate of ultrahigh energy nuclei in the immediate environment of young fast-rotating pulsars, JCAP 1508 (2015) 026 [1503.07907].

[12] K. Ptitsyna and A. Neronov, Particle acceleration in the vacuum gaps in black hole magnetospheres, Astron. Astrophys. 593 (2016) A8 [1510.04023].

[13] J. Heinze, D. Boncioli, M. Bustamante and W. Winter, Cosmogenic Neutrinos Challenge the Cosmic Ray Proton Dip Model, Astrophys. J. 825 (2016) 122 [1512. 05988].

[14] R. C. Gilmore, R. S. Somerville, J. R. Primack and A. Dominguez, Semi-analytic modeling of the EBL and consequences for extragalactic gamma-ray spectra, Mon. Not. Roy. Astron. Soc. 422 (2012) $3189[1104.0671]$.

[15] M. De Domenico, M. Settimo, S. Riggi and E. Bertin, Reinterpreting the development of extensive air showers initiated by nuclei and photons, JCAP 1307 (2013) 050 [1305.2331].

[16] T. Pierog et al., First results of fast one-dimensional hybrid simulation of EAS using CONEX, Nucl. Phys. Proc. Suppl. 151 (2006) 159 [astro-ph/ 0411260 ].

[17] Pierre Auger Collaboration collaboration, Depth of Maximum of Air-Shower Profiles at the Pierre Auger Observatory: Composition Implications, Phys.Rev.D (2014) [1409.5083].

[18] PierRe Auger collaboration, Combined fit of spectrum and composition data as measured by the Pierre Auger Observatory, JCAP 1704 (2017) 038 [1612 . 07155].

[19] R. Alves Batista, A. Dundovic, M. Erdmann, K.-H. Kampert, D. Kuempel, G. MÃijller et al., CRPropa 3 - a Public Astrophysical Simulation Framework for Propagating Extraterrestrial Ultra-High Energy Particles, JCAP 1605 (2016) 038 [1603.07142].

[20] B. Katz, R. Budnik and E. Waxman, The energy production rate \&amp; the generation spectrum of UHECRs, JCAP 0903 (2009) 020 [0811.3759].

[21] E. Waxman, Cosmological origin for cosmic rays above 10**19-eV, Astrophys. J. 452 (1995) L1 [astro-ph/9508037].

[22] J. N. Bahcall and E. Waxman, Has the GZK cutoff been discovered?, Phys. Lett. B556 (2003) 1 [hep-ph/0206217].

[23] V. Berezinsky, Propagation and origin of ultra high-energy cosmic rays, Advances in Space Research 41 (2008) 2071.

[24] PIERRE Auger collaboration, The Pierre Auger Observatory Upgrade - Preliminary Design Report, 1604.03637. 